\title{
Erratum to: Comprehensive gene panels provide advantages over clinical exome sequencing for Mendelian diseases
}

Saudi Mendeliome Group*

\section{Erratum}

Unfortunately, the original version of this article [1] contained a mistake. In the Acknowledgement section of the article the author Saeed Bohlega was incorrectly spelt as Saeed Boholega. We apologise for this error. The author's name has been included correctly in the list below:

\section{Acknowledgement}

This study was supported by a research grant from King Salman Center for Disability Research (FSA) as well as the Saudi Human Genome Project initiative, KACST.Saudi Mendeliome Group (alphabetical order): Abdulwahab, Firdous; Abouelhoda, Mohamed; Abouthuraya, Rula; Abumansour, Iman; Ahmed, Syed O.; Al Rubeaan, Khalid; Al Tassan, Nada; AlAbdulaziz, Basma; AlAbdulrahman, Khalid; Alamer, FH; Alazami, Anas; Al-Baik, Lina A.; Aldahmesh, Mohammed; Al-Dhekri, Hasan; AlDusery, Haya; Algazlan, Sulaiman; Al-Ghonaium, Abdulaziz; Alhamed, Mohammed; Alhashem, Amal; Alhissi, Safa Ahmed; AlIssa, Abdulelah; Aljurf, Mahmoud D.; Alkuraya, Fowzan S; Alkuraya, Hisham; Allam, Rabab; Almasharawi, Iman J; Almoisheer, Agaadir; AlMostafa, Abeer; Al-Mousa, Hamoud; Al-Muhsen, Saleh; Almutairy, Eid A; Alnader, Noukha; AlNaqeb, Dhekra; ALOtaibi, AB; Alotibi, Afaf; Al-Qattan, Sarah; Al-Saud, Bandar; Al-Saud, Haya; Alshammari, M; Alsharif, Hadeel; Alsheikh, Abdulmoneem H; Al-Sulaiman, Ayman; Altamimi, AS; Al-Tayeb, Hamsa; Alwadaee, SM; Al-Younes, B; Alzahrani, Fatima; Anazi, Shamsa; Arnaout, Rand; Bashiri, Fahad; Binamer, Yousef; Binhumaid, FS; Bohlega, Saeed; Broering, Dieter; Burdelski, Martin; Dasouki, Majed Jamil; Dzimiri, Nduna Francis; Elamin, Tanziel; El-Kalioby, Mohamed; Elsiesy, Hussien; Faqeih, Eissa; Faquih, Tariq; Hagos, Samya; Hagr, Abdulrahman A; Hashem, Mais; Hawwari,

\footnotetext{
* Correspondence: falkuraya@kfshrc.edu.sa

${ }^{1}$ Saudi Human Genome Project, King Abdulaziz City for Science and Technology, Riyadh, Saudi Arabia
}

Abbas; Hazzaa, Selwa; Ibrahim, Niema; Imtiaz, Faiqa; Jabr, Amal; Kattan, Rana; Kaya, Namik; Kentab, Amal; Khalil, Dania; Khan, Arif O; Khier, Omnia; Meyer, Brian; Mohamed, Jawahir; Monies, Dorota; Muiya, Paul N.; Murad, Hatem; Naim, EA; Owaidah, Tarek; Patel, Nisha; Ramzan, Khushnooda; Salih, Mustafa A; Shagrani, Mohammad; Shaheen, Ranad; Shamseldin, Hanan; Sogaty, Sameera; Subhani, Shazia; Taibah, Khalid; Wakil, Salma M.

Received: 7 October 2015 Accepted: 7 October 2015

Published online: 13 October 2015

\section{Reference}

1. Saudi Mendeliome Group. Comprehensive gene panels provide advantages over clinical exome sequencing for Mendelian diseases. Genome Biol. $2015 ; 16: 134$

\section{Submit your next manuscript to BioMed Central and take full advantage of: \\ - Convenient online submission \\ - Thorough peer review \\ - No space constraints or color figure charges \\ - Immediate publication on acceptance \\ - Inclusion in PubMed, CAS, Scopus and Google Scholar \\ - Research which is freely available for redistribution \\ Submit your manuscript at www.biomedcentral.com/submit}

\section{Biomed Central}

(c) 2015 Saudi Mendeliome Group. Open Access This article is distributed under the terms of the Creative Commons Attribution 4.0 International License (http://creativecommons.org/licenses/by/4.0/), which permits unrestricted use, distribution, and reproduction in any medium, provided you give appropriate credit to the original author(s) and the source, provide a link to the Creative Commons license, and indicate if changes were made. The Creative Commons Public Domain Dedication waiver (http://creativecommons.org/publicdomain/zero/1.0/) applies to the data made available in this article, unless otherwise stated. 